\title{
Massive intravascular hemolysis associated with Clostridium perfringens bacteremia
}

\author{
Giovanni Poletti ${ }^{1}$, Evita Massari ${ }^{1}$, Andrea Fabbri ${ }^{2}$, Marta Monti ${ }^{1}$, Marco Rosetti ${ }^{1}$, Vittorio Sambri ${ }^{3,4}$ \\ ${ }^{1}$ Clinical Pathology Unit, Hub Laboratory, AUSL della Romagna, Cesena, ${ }^{2}$ Emergency Department, Presidio Ospedaliero \\ Morgagni-Pierantoni, AUSL della Romagna, Forli, ${ }^{3}$ Microbiology Unit, Hub Laboratory, AUSL della Romagna, Cesena, ${ }^{4}$ DIMES, \\ University of Bologna, Bologna, Italy
}

Received on November 10, 2020; Revised on December 1, 2020; Accepted on January 19, 2021

Correspondence to Giovanni Poletti, M.D., Clinical Pathology Unit, Hub Laboratory, AUSL della Romagna, Cesena 47521, Italy, E-mail: giovanni.poletti@auslromagna.it
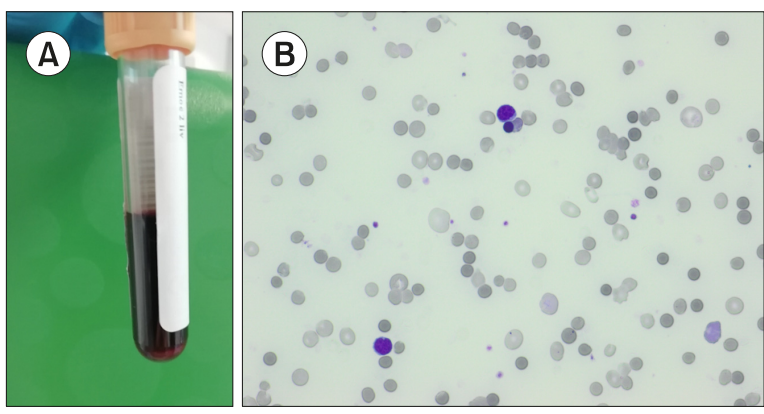

A 64-year-old woman with fever and weakness was recently administered the first course of chemotherapy for acute myeloid leukemia. Tests of a blood sample at our emergency laboratory showed the following: red blood cell (RBC) count, $0.91 \times 10^{12} / \mathrm{L}$; hematocrit, 0.07 (L/L); hemoglobin concentration, $3.6 \mathrm{~g} / \mathrm{dL}$; mean corpuscular volume, $76.9 \mathrm{fL}$; mean corpuscular hemoglobin, $39.6 \mathrm{pg}$; mean corpuscular hemoglobin concentration, $514 \mathrm{~g} / \mathrm{L}$; RBC distribution width, 16.8\%; RBC distribution width with standard deviation, $55.8 \mathrm{fL}$; white blood cell count, $0.87 \times 10^{9} / \mathrm{L}$; platelet count, $28 \times 10^{9} / \mathrm{L}$; nucleated RBC count, $0.56 \times 10^{9} / \mathrm{L}$; and reticulocyte count, $181.2 \times 10^{9} / \mathrm{L}$. Plasma and serum samples appeared grossly hemolyzed, and all biochemical tests failed (A). Blood film showed many microspherocytes, nucleated RBCs, rare neutrophils, and the absence of blasts (B). Numerous spherocytes are observed in hereditary spherocytosis, immune hemolytic anemia, burns, toxic damage, and infections. Clostridium perfringens $\alpha$ toxin hydrolyzes phospholipids in RBC membranes, causing spherocytosis and hemolysis. Herein, a negative Coombs' test result and C. perfringens identification in blood cultures led to accurate diagnosis. Prompt concentrated RBC transfusions and vancomycin antibiotic therapy avoided an otherwise likely and rapidly fatal outcome. Massive hemolysis, masking critical care settings, may be incorrectly considered an error during sample collection. Blood smear revisions aid differential diagnosis. 